\title{
Thyroglobulin Levels in Patients who have Undergone Hemithyroidectomy for Differentiated Thyroid Cancer. An Assessment of Levels and Trends at a Tertiary Referral Centre
}

\author{
Ashwini Munnagi ${ }^{1}$ Vijay Pillai ${ }^{1} \quad$ R. Vidhya Bushan ${ }^{1}$ Vivek Shetty ${ }^{1}$ Narayana Subramaniam ${ }^{1}$ \\ K. S. Shivaprasad ${ }^{2}$ Kranti Khadilkar ${ }^{2}$ Basavaraj G. Sooragonda ${ }^{2}$ Akhila Lakhsmikantha ${ }^{3}$ \\ Pobbisetty Radhakrishnagupta Rekha ${ }^{3}$ Shaesta Naseem Zaidi ${ }^{3}$ Nishtha Batra ${ }^{3}$ Subramanian Kannan ${ }^{2}$ \\ 1 Department of Head and Neck Surgical Oncology, Narayana \\ Hrudhayalaya Hospitals, Bangalore, Karnataka, India \\ ${ }^{2}$ Department of Endocrinology, Diabetes and Metabolism, Narayana \\ Hrudhayalaya Hospitals, Bangalore, Karnataka, India \\ ${ }^{3}$ Department of Pathology and Laboratory Medicine, Narayana \\ Address for correspondence Subramanian Kannan, MD, AB, \\ Department of Endocrinology, Diabetes and Metabolism, Mazumdar \\ Shaw Medical Center, Narayana Health City, 258/A Bommasandra \\ Industrial Area, Hosur Road, Bangalore-560099, Karnataka, India \\ (e-mail: subramanian.kannan@gmail.com).
} Hrudhayalaya Hospitals, Bangalore, Karnataka, India

\section{South Asian J Cancer 2022;11(1):52-57.}

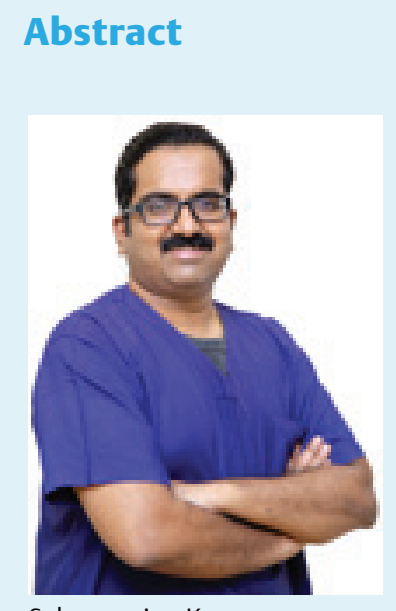

Subramanian Kannan

\section{Keywords}

- serum thyroglobulin

- hemithyroidectomy

- lobectomy

- Tg levels

- differentiated thyroid cancer
Serum thyroglobulin $(\mathrm{Tg})$ and thyroglobulin antibody $(\mathrm{TgAb})$ levels are used to monitor patients with differentiated thyroid cancer (DTC) after total thyroidectomy with or without radioiodine (RAI) ablation. However, they are also measured in patients who are treated with thyroid lobectomy ( $\mathrm{TL}$ )/hemithyroidectomy $(\mathrm{HT})$. Data on the levels of $\mathrm{Tg}$ and its trend in those undergoing TL/HT is sparse in India. We reviewed retrospective data of DTC patients who underwent TL/HT and were followed-up with postoperative $\mathrm{Tg}$ levels between 2015 and 2020. Out of 247 patients, 17 had undergone either TL or HT, which included papillary thyroid cancer $(n=12)$, follicular thyroid cancer $(n=4)$, and noninvasive follicular thyroid neoplasm with papillary-like nuclear features (NIFTP) in 1 patient. All patients with DTC had tumor size $<4 \mathrm{~cm}$ (T1/2, clinical N0, Mx). The median follow-up was 15 months (range, 1-125) and the median $\mathrm{Tg}$ level was $7.5 \mathrm{ng} / \mathrm{mL}$ (interquartile range [IQR]; 3.6, 7.5) and ranged from 0.9 to $36.7 \mathrm{ng} / \mathrm{mL}$. The median thyroid-stimulating hormone (TSH) level was $2.03 \mathrm{IU} / \mathrm{L}$ (IQR; 1.21 , 3.59) and it ranged from 0.05 to $8.54 \mathrm{IU} / \mathrm{L}$. As of last follow-up, none of them underwent completion thyroidectomy; however, eight patients had a decline in $\mathrm{Tg}$ ranging from 8 to $64 \%$, four patients had increase in $\mathrm{Tg}$ ranging from 14 to $145 \%$, three patients had stable $\mathrm{Tg}$, and one of them had an increase in TgAb titers. As per American Thyroid Association (ATA) response-to-treatment category, six patients had indeterminate response, five patients had biochemical incomplete response, four patients had excellent response, and two did not have follow-up Tg and TgAb levels. While absolute values of Tg were well below $30 \mathrm{ng} / \mathrm{mL}$ in almost all patients with $\mathrm{HT} / \mathrm{TL}$, the $\mathrm{Tg}$ trends were difficult to predict, and only $23 \%$ of patients were able to satisfy the criteria for "excellent response" on follow-up. We suggest keeping this factor in mind in follow-up and while counselling for HT in patients with low-risk DTC.
DOI https://doi.org/10.1055/s-0041-1733315 ISSN 2278-330X

How to cite this article: Munnagi A, Pillai V, Bushan R. V et al. Thyroglobulin Levels in Patients who have Undergone Hemithyroidectomy for Differentiated Thyroid Cancer. An Assessment of Levels and Trends at a Tertiary Referral Centre South Asian J Cancer 2022;11(1):52-57.
(C) 2021. MedIntel Services Pvt Ltd. All rights reserved.

This is an open access article published by Thieme under the terms of the Creative Commons Attribution-NonDerivative-NonCommercial-License, permitting copying and reproduction so long as the original work is given appropriate credit. Contents may not be used for commercial purposes, or adapted, remixed transformed or built upon. (https://creativecommons.org/licenses/by-nc-nd/ 4.0/)

Thieme Medical and Scientific Publishers Pvt. Ltd., A-12, 2nd Floor, Sector 2, Noida-201301 UP, India 


\section{Background}

The 2015 American Thyroid Association (ATA) guidelines ${ }^{1}$ recommend thyroid lobectomy (TL) as the initial procedure for patients with DTC $\leq 1 \mathrm{~cm}$ (if surgery is chosen, instead of active surveillance), no extrathyroidal extension (ETE), no clinical lymph nodal metastasis, no prior history of head and neck irradiation, and no familial history of thyroid carcinoma. TL is also recommended for patients with DTC $>1 \mathrm{~cm}$ and $\leq 4 \mathrm{~cm}$ (without ETE and clinical evidence of lymph node [LN] metastasis), low-risk papillary thyroid cancer (PTC), and follicular thyroid carcinoma (FTC). ${ }^{1}$ Neck ultrasonography and serial serum thyroglobulin (Tg) measurements are more preferred as follow-up tools than whole-body radioiodine (RAI) scans. ${ }^{1}$ The NCCN guidelines recommend measuring $\mathrm{Tg}$ and thyroglobulin antibody (TgAb) 6 to 12 weeks after initial surgery and assessing trend patterns in TL patients. While the ATA response-to-treatment classification is focused on patients undergoing total thyroidectomy (TT) with or without RAI remnant ablation, it can also be applied to patients with DTC undergoing TL or hemithyroidectomy (HT) without RAI remnant ablation, by using modified definitions of the response-to-therapy categories ( - Table 1 ). ${ }^{3}$ Recent studies showed that the response-to-treatment system was effective for modifying the initial risk estimates of structural recurrence/persistence and provided valuable information for predicting structural recurrence even in TL patients. Although specific criteria for distinguishing normal residual thyroid tissue from persistent or recurrent thyroid cancer have not been defined, most patients with an excellent response have a serum $\mathrm{Tg}$ level $<30 \mathrm{ng} / \mathrm{mL}$, undetectable $\operatorname{TgAb}$, and negative imaging (mostly a normal ultrasound of the neck) (-Table 1). A rising value of Tg over time is suspicious for thyroid cancer (or growth of normal thyroid tissue). Those with detectable $\mathrm{TgAb}$ would be considered to be "indeterminate" ATA response to treatment and those with rising titers of $\mathrm{Tg}$ and/or TgAb antibodies are regarded as "biochemical incomplete" response (-Table 1 ). Any struc-

Table 1 Response to treatment definitions after initial TL3

\begin{tabular}{|l|l|}
\hline Excellent response & $\begin{array}{l}\text { Stable, nonstimulated Tg level }<30 \\
\text { ng/mL and undetectable TgAb and } \\
\text { negative imaging }\end{array}$ \\
\hline $\begin{array}{l}\text { Biochemical incomplete } \\
\text { response }\end{array}$ & $\begin{array}{l}\text { Nonstimulated Tg level }>30 \mathrm{ng} / \mathrm{mL} \\
\text { or } \\
\text { increasing Tg level values over time } \\
\text { with similar TSH levels } \\
\text { or } \\
\text { increasing TgAb levels } \\
\text { and } \\
\text { negative imaging }\end{array}$ \\
\hline $\begin{array}{l}\text { Structural incomplete } \\
\text { response }\end{array}$ & $\begin{array}{l}\text { Structural or functional evidence of } \\
\text { disease regardless of Tg or TgAb }\end{array}$ \\
\hline Indeterminate response & $\begin{array}{l}\text { Nonspecific findings on imaging } \\
\text { studies } \\
\text { or } \\
\text { TgAb levels stable or declining in the } \\
\text { absence of structural or functional } \\
\text { disease }\end{array}$ \\
\hline
\end{tabular}

Abbreviations: $\mathrm{Tg}$, thyroglobulin; $\mathrm{TgAb}$, thyroglobulin antibody; TL, thyroid lobectomy; TSH, thyroid-stimulating hormone. tural evidence of cancer detected either by ultrasound or cross-sectional or functional imaging is regarded as "structurally incomplete response," regardless of the levels of Tg or TgAb. Data on Tg levels and trends of Tg in patients who have undergone TL/HT is limited from India. In this study, we reviewed and assessed the $\mathrm{Tg}$ and $\operatorname{TgAb}$ levels during the follow-up in patients who underwent HT.

\section{Materials and Methods}

We reviewed retrospective data of patients with thyroid cancer who had followed-up at Narayana Hrudayalaya Hospitals, Bangalore, between 2015 and 2020. We included only patients who underwent TL/HT either in our institution or elsewhere for DTC. Decisions on the extent of surgery were at the discretion of the treating physician, with consideration for patient preference. Patients with a minimum follow-up period of 3 months and who had sufficient data on Tg and TgAb levels during follow-up were included. Any patients with histopathological diagnoses other than FTC/PTC, or with cervical lymph node metastases, or distant metastases were excluded from the analysis. All patients underwent Tg and TgAb during follow-up, using immunochemiluminescent assay with Roche Cobas C311 analyzer (Roche Diagnostics GmbH, Mannheim, Germany) from 2010 to September 2017 with lower limit of detection of $0.4 \mathrm{ng} / \mathrm{mL}$ and ADVIA Centaur xPT immunoassay system (Siemens Healthcare Diagnostics Inc., Tarrytown, NY, USA) from October 2017 to date with lower limit of detection of $1.6 \mathrm{ng} / \mathrm{mL}$. TgAb was simultaneously assayed with normal titers between 0 to $115 \mathrm{IU} / \mathrm{mL}$ (Roche Cobas C311) and 0 to $60 \mathrm{IU} / \mathrm{mL}$ (ADVIA Centaur XPT). Disease stage was determined based on the eighth edition of the American Joint Committee on Cancer (AJCC) staging system, and response to treatment was categorized using ATA response to treatment. ${ }^{1,6}$ The follow-up period was defined as the time between removal of the first lobe and the last documented laboratory tests for thyroid-stimulating hormone (TSH), Tg and TgAb. Patients received thyroxine replacement to maintain TSH levels between 0.5 to $2.5 \mathrm{IU} / \mathrm{L}$ on follow-up, and Tg levels on comparable and similar levels of TSH were analyzed.

\section{Statistical Methods}

Data was analyzed using SPSS (IBM SPSS Statistics for Windows, Version 20.0. Armonk, NY: IBM Corp) for descriptive and inferential statistics. Categorical data are presented in terms of number and percentage, whereas continuous data are presented as mean and standard deviation (SD) or median and range.

\section{Results}

A total of 247 charts were reviewed, out of whom 17 patients have undergone either TL/HT. There were 13 females, and the mean $\pm \mathrm{SD}$ (range) age of these patients was $35 \pm 12$ (16-59) years. The complete details of basic and clinical details of the 
HT patients are presented in -Table 2. Among the 17 patients, 12 had PTC (10 classic and 2 follicular variant), 4 had FTC, and one had noninvasive follicular thyroid neoplasm with papillary-like nuclear features (NIFTP). All the PTC and FTC were less than $4 \mathrm{~cm}$ (tumor stage T1 or T2 with clinical N0). Fourteen patients were on thyroxine replacement therapy. After a median follow-up of 15 months (range, 1-125), the median $\mathrm{Tg}$ level was $7.5 \mathrm{ng} / \mathrm{mL}$ (interquartile range [IQR]; 3.6, 7.5) and it ranges from 0.9 to $36.7 \mathrm{ng} / \mathrm{mL}$. The median TSH level was $2.03 \mathrm{IU} / \mathrm{L}(\mathrm{IQR} ; 1.21,3.59)$ and it ranges from 0.05 to $8.54 \mathrm{IU} / \mathrm{L}$. At the end of follow-up, 8 patients had decline in $\mathrm{Tg}$ (ranging from $1-20 \mathrm{ng} / \mathrm{mL}$; range 7.7-63.5\%) with 7 patients having undetectable $\operatorname{TgAb}$ and 2 patients having $\mathrm{TgAb}$ in the normal range $(18-20 \mathrm{IU} / \mathrm{mL})$ and 4 patients had increase in $\operatorname{Tg}(1,3,3.5$, and 11 , respectively; range 13.6-144.7\%), and all these patients had undetectable TgAb antibodies; 3 patients had stable $\mathrm{Tg}$, with two patients having undetectable antibodies and one of them having three-fold increase in TgAb titers. Two patients had followed-up less than 6 months and did not have a follow-up $\operatorname{Tg}$ or $\operatorname{TgAb}$. No patient had suspicious nodules in the opposite lobe or suspicious lymph nodes on ultrasound of the neck. No patient underwent completion thyroidectomy as of current follow-up information. As per ATA response to treatment category, 6 patients had indeterminate response (35\%), 5 patients had biochemical incomplete response (29\%), and 4 patients had excellent response (23\%).

\section{Discussion}

With the increasing prevalence of low-risk DTC among patients, individualized treatment with emphasis on riskadapted approach is recommended. ${ }^{7}$ HT is adequate in the management of DTC patients, if a $1 \%$ risk of recurrence to the remnant thyroid is accepted. ${ }^{6}$ This is a more conservative approach, and a follow-up strategy has been suggested in recent literature, which shows that the therapeutic efficacy of TL is comparable with that of TT. ${ }^{1}$ It has been recommended that regular neck ultrasound and serum Tg measurements are essential in the follow-up of these patients. ${ }^{5}$ The prognostic value of Tg following TL for DTC has gained interest in recent years as part of a paradigm shift aimed at risk-adapted, individualized therapy and follow-up. Vaismann et al suggested that in the follow-up of lobectomy patients, any increase or decrease in serum Tg levels by more than $20 \%$ in two consecutive measurements was clinically significant, and they suggested that stable or decreased serum $\mathrm{Tg}$ levels was associated with a lower probability of recurrence. ${ }^{4}$ A recent study from Israel find that serum $\mathrm{Tg}$ used independently is of limited value for predicting or detecting disease recurrence following $\mathrm{TL}^{8}{ }^{8}$ While $\mathrm{Tg}$ is a well-established component of the 2015 ATA response-totreatment system following TT with or without iodine ablation, its interpretation following TL/HT is challenging. This is due to $\mathrm{Tg}$ produced by the remaining lobe, which shows considerable interpatient variability due to lobe size, TSH levels, lymphocytic thyroiditis, thyroid nodules, iodine status, and other factors. If a lobe produces about half the $\mathrm{Tg}$ produced by the intact thyroid gland (range $20-60 \mathrm{ng} / \mathrm{mL}$ ), then small amounts of $\mathrm{Tg}$ produced by persistent/recurrent cancer tissue might go unnoticed within the total amount measured. Despite this challenge, Momesso et al suggested based on neck ultrasound and basal Tg levels, a Tg threshold of $<30 \mathrm{ng} / \mathrm{mL}$ in the absence of antibodies for the definition of excellent response to therapy. ${ }^{3,5}$ But, Park et al reported that serum Tg levels in TL/HT patients increased gradually without definite evidence of disease recurrence during the follow-up, suggesting a compensatory increase of the remnant thyroid tissue after lobectomy, which may have contributed to the increase seen in the serum Tg levels. They also found that decreased or stable serum $\mathrm{Tg}$ levels did not guarantee a lower probability of recurrence. ${ }^{9}$ Similarly, Ritter et al showed that there was an overlap between $\mathrm{Tg}$ levels in patients who underwent TL/HT and those who had disease recurrence $(22.5 \pm 22.3 \mathrm{ng} / \mathrm{mL})$ and those who did not (11.3 $\pm 13.8 \mathrm{ng} / \mathrm{ml}$ ), with no threshold that could distinguish between the two groups. ${ }^{8}$ They also noted that, although in half the cases with recurrence, there was a steady rise in $\mathrm{Tg}$ levels during follow-up, this was also observed in $34 \%$ of the nonrecurrent group. They concluded that both basal Tg levels and increasing $\mathrm{Tg}$ levels during follow-up were not indicators of disease recurrence.

There are some practical issues applying the $\mathrm{Tg}$ cutoff levels to decide on ATA response-to-treatment classification. The presence of detectable "normal level" titers of TgAb can also affect the level of Tg and this is most relevant when $\mathrm{TgAb}$ titers go down and Tg levels increase. The relevance of these changes are unclear. Rising TgAb titers also pose another difficulty to the clinicians. The biggest challenge of HT is the inability to accurately stage the disease, in view of not doing a RAI whole body scan. In our study, the proportion of patients achieving excellent response to treatment was better (30\%) in the TT cohort compared with only $23 \%$ of those with TL/HT cohort. Most patients with HT/TL have detectable but "normal range" TgAb and some indeterminate ultrasound lesions which place them in the "indeterminate category." We feel that interpretation of Tg levels in HT is complex and leads to difficulties in counselling and "comforting" the patient. We are limited by a small sample of retrospective observation, and also have not correlated $\mathrm{Tg}$ levels with presence of nodules in the contralateral lobe or background of thyroiditis and iodine status. However, we were able to show that the levels of Tg after HT were well within the accepted range of $<30 \mathrm{ng} / \mathrm{mL}$ as quoted in the literature, however the trend of $\mathrm{Tg}$ and impact of $\mathrm{TgAb}$ on $\mathrm{Tg}$ levels makes interpretation complex on follow-up.

\section{Conclusion}

At a median follow-up of 15 months in patients who underwent TL/HT, the median Tg level was $7.5 \mathrm{ng} / \mathrm{mL}$, and Tg levels were either stable or decreased in $65 \%$ of the patients. While absolute values of $\mathrm{Tg}$ are well below $30 \mathrm{ng} / \mathrm{mL}$ in almost all patients with HT/TL, the Tg trends are difficult to predict and only $23 \%$ of patients were able to satisfy the criteria for "excellent response" on follow-up. While carefully selected 


\begin{tabular}{|c|c|c|c|c|c|c|c|c|c|c|c|}
\hline 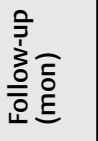 & - & $\nexists$ & \& & 6 & $\infty$ & $\stackrel{n}{\sim}$ & 0 & $m$ & - & 우 & $\stackrel{n}{\sim}$ \\
\hline 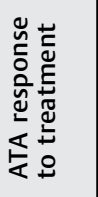 & 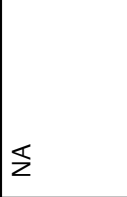 & 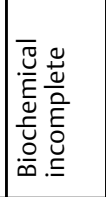 & 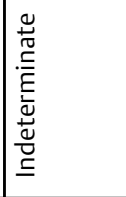 & 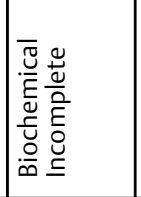 & 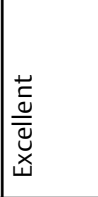 & 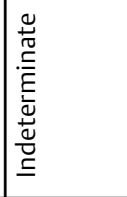 & $\S$ & 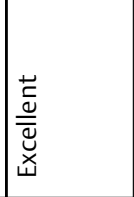 & 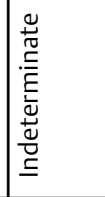 & 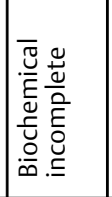 & 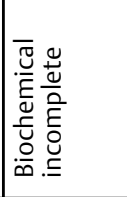 \\
\hline 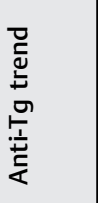 & $\bar{z}$ & 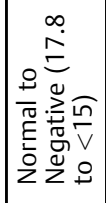 & 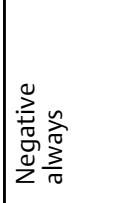 & 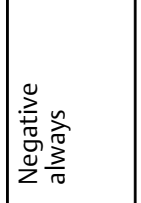 & 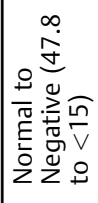 & 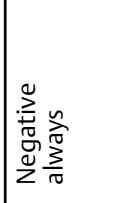 & $\S$ & 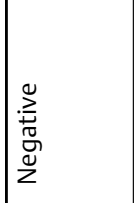 & 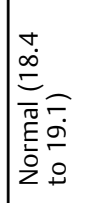 & 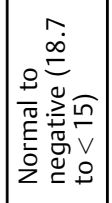 & 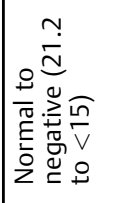 \\
\hline 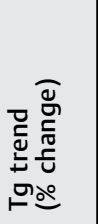 & $\Sigma$ & 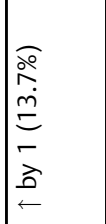 & 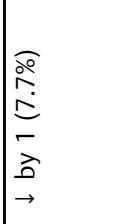 & 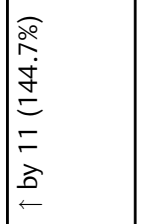 & $\uparrow$ & 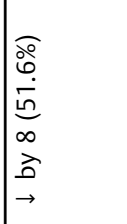 & $\Sigma$ & 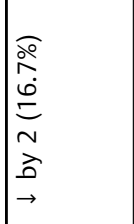 & 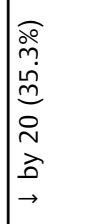 & 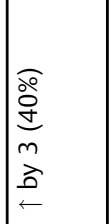 & 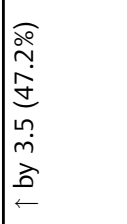 \\
\hline 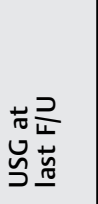 & 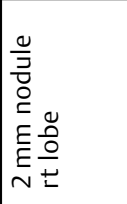 & \begin{tabular}{|l}
$\overline{\widetilde{\pi}}$ \\
$\tilde{E}$ \\
$\hat{z}$
\end{tabular} & 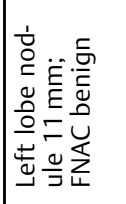 & 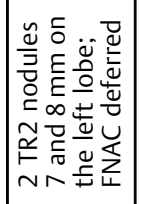 & \begin{tabular}{|l}
$\overline{\widetilde{T}}$ \\
$\stackrel{\underline{\xi}}{0}$ \\
$z$
\end{tabular} & 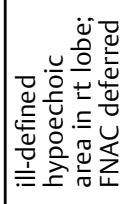 & \begin{tabular}{|l}
$\overline{\widetilde{\pi}}$ \\
$\stackrel{\underline{E}}{0}$ \\
$\dot{z}$
\end{tabular} & \begin{tabular}{|l}
$\overline{\widetilde{\pi}}$ \\
$\tilde{E}$ \\
$\hat{z}$
\end{tabular} & \begin{tabular}{|l}
$\overline{\widetilde{\pi}}$ \\
$\underline{\tilde{E}}$ \\
$\dot{z}$
\end{tabular} & 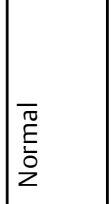 & \begin{tabular}{|l}
$\overline{\widetilde{T}}$ \\
$\stackrel{\underline{E}}{0}$ \\
$\dot{z}$
\end{tabular} \\
\hline 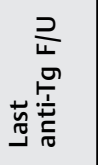 & 6 & 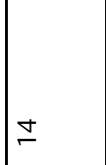 & 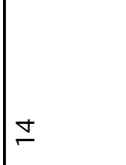 & 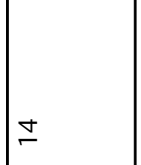 & 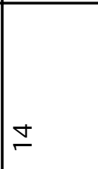 & a & 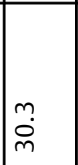 & $\underset{\square}{ \pm}$ & $\bar{g}$ & $\underset{\square}{ \pm}$ & 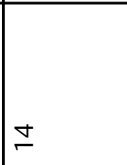 \\
\hline 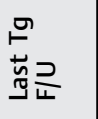 & $\stackrel{\sim}{\stackrel{2}{\sim}}$ & $\underset{\infty}{m}$ & $\stackrel{?}{=}$ & $\begin{array}{l}0 \\
\infty \\
\infty\end{array}$ & $\stackrel{N}{r}$ & $\stackrel{n ?}{\sim}$ & $\stackrel{\varphi}{\stackrel{0}{*}}$ & $\stackrel{\circ}{\circ}$ & $\hat{\grave{n}}$ & 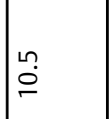 & $\stackrel{\infty}{\curvearrowright}$ \\
\hline 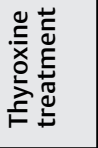 & $\stackrel{\check{y}}{\searrow}$ & $\stackrel{\check{y}}{\succ}$ & i & z) & $\stackrel{\check{\varpi}}{\rightleftharpoons}$ & $\stackrel{\check{\Xi}}{\succ}$ & $\stackrel{\check{y}}{\succ}$ & $\stackrel{\check{y}}{\rightleftharpoons}$ & $\stackrel{\check{\nu}}{\rightleftharpoons}$ & ż & $\stackrel{\check{\varphi}}{\rightleftharpoons}$ \\
\hline 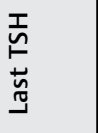 & 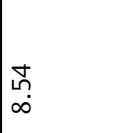 & 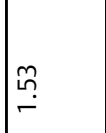 & in & 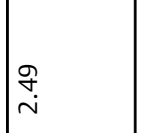 & $\stackrel{\infty}{\stackrel{\infty}{m}}$ & $\mid \begin{array}{r}\tilde{\sim} \\
\stackrel{\sim}{\sim}\end{array}$ & 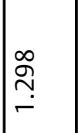 & $\stackrel{m}{i}$ & $\stackrel{\leftrightarrow}{m}$ & $\underset{\sim}{\stackrel{J}{m}}$ & 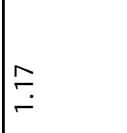 \\
\hline 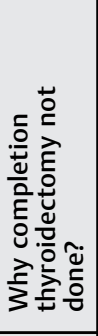 & 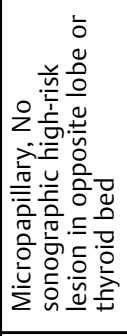 & 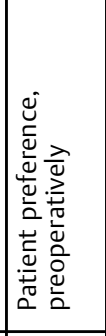 & 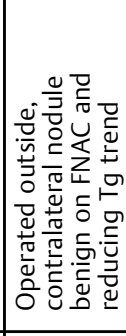 & 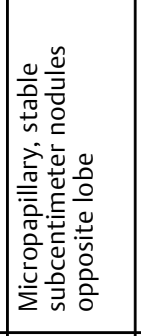 & 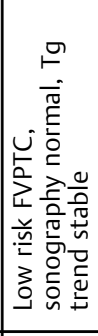 & 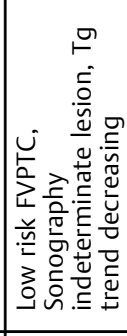 & 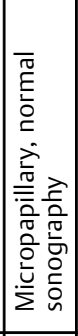 & 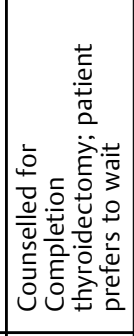 & 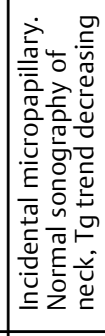 & 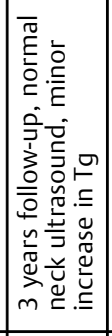 & 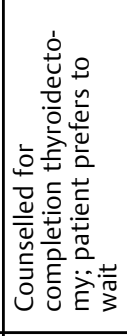 \\
\hline$\vdash$ & - & $\stackrel{\circ}{\circ}$ & - & 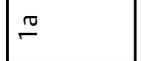 & $\sim$ & $\sim$ & $\stackrel{0}{\circ}$ & $\sim$ & $\simeq$ & $\stackrel{P}{\circ}$ & N \\
\hline 㠉 & $\breve{a}$ & 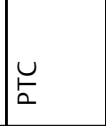 & 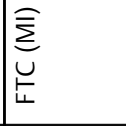 & $\breve{\iota}$ & 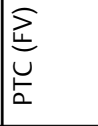 & 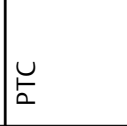 & 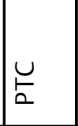 & 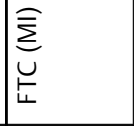 & 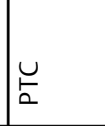 & $\breve{\iota}$ & $\mid \begin{array}{l}\overline{\bar{\Sigma}} \\
u \\
\underline{u}\end{array}$ \\
\hline 这 & $\tilde{m}$ & શ & I & 迤 & 方 & $\stackrel{0}{2}$ & & $\approx$ & |స & 品 & $\hat{m}$ \\
\hline 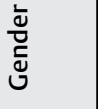 & 4 & $\sqcup$ & 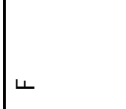 & $\Sigma$ & $\Sigma$ & 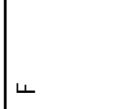 & L & 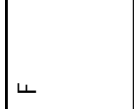 & ᄂ & $\Sigma$ & 4 \\
\hline
\end{tabular}


56 Hemithyroidectomy for Differentiated Thyroid Cancer Munnagi et al.

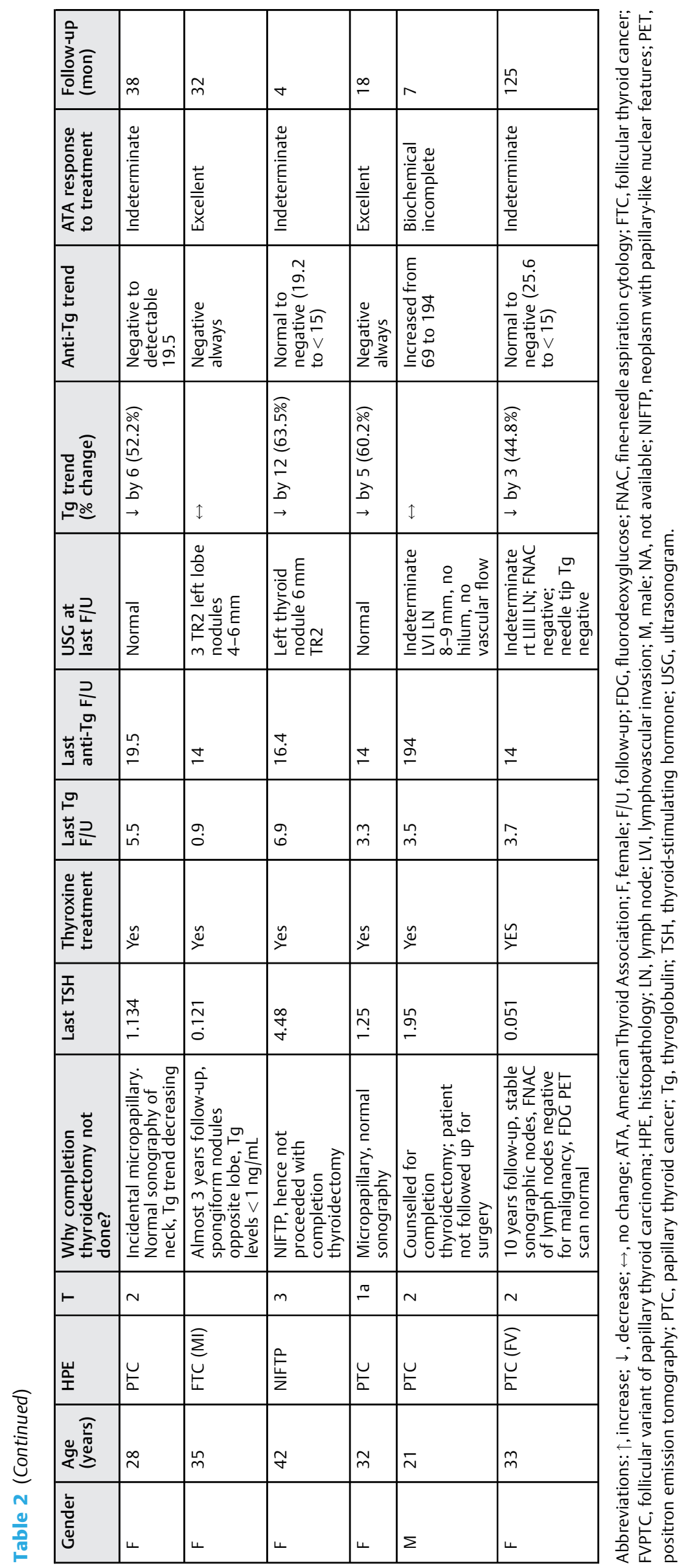

South Asian Journal of Cancer Vol. 11 No. 1/2022 @ 2021. MedIntel Services Pvt Ltd. All rights reserved. 
patients with "low-risk" DTC can be followed up after TL/HT with serial $\mathrm{Tg} / \mathrm{TgAb}$ and neck sonography, one needs to understand the complexity in interpretation of $\mathrm{Tg} / \mathrm{TgAb}$ levels on follow-up and consider this aspect while recommending HT in "low-risk" DTC.

\section{Ethical Approval}

This study was approved by an independent institutional review board and ethics committee (Ref number: NHH/AEC-CL-2016-090).

\section{Funding Statement}

This study did not receive any specific fund from a private or government or nonprofit funding agencies.

\section{Conflicts of Interest}

None declare.

Acknowledgment

None.

\section{References}

1 Haugen BR, Alexander EK, Bible KC, et al. 2015 American Thyroid Association Management Guidelines for Adult Patients with Thyroid Nodules and Differentiated Thyroid Cancer: The Ameri- can Thyroid Association Guidelines Task Force on Thyroid Nodules and Differentiated Thyroid Cancer. Thyroid 2016;26(01):1-133

2 Haddad RI, Nasr C, Bischoff L, et al. NCCN Guidelines Insights: Thyroid Carcinoma, Version 2.2018. J Natl Compr Canc Netw 2018;16(12):1429-1440

3 Momesso DP, Tuttle RM. Update on differentiated thyroid cancer staging. Endocrinol Metab Clin North Am 2014;43(02):401-421

4 Vaisman F, Momesso D, Bulzico DA, et al. Thyroid lobectomy is associated with excellent clinical outcomes in properly selected differentiated thyroid cancer patients with primary tumors greater than $1 \mathrm{~cm}$. J Thyroid Res 2013;2013:398194

5 Momesso DP, Vaisman F, Yang SP, et al. Dynamic risk stratification in patients with differentiated thyroid cancer treated without radioactive iodine. J Clin Endocrinol Metab 2016;101(07): 2692-2700

6 Shaha AR, Migliacci JC, Nixon IJ, et al. Stage migration with the new American Joint Committee on Cancer (AJCC) staging system (8th edition) for differentiated thyroid cancer. Surgery 2019;165 (06): 11

7 Adam MA, Pura J, Gu L, et al. Extent of surgery for papillary thyroid cancer is not associated with survival: an analysis of 61,775 patients. Ann Surg 2014;260(04):601-605, discussion 605-607

8 Ritter A, Mizrachi A, Bachar G, et al. detecting recurrence following lobectomy for thyroid cancer: role of thyroglobulin and thyroglobulin antibodies. J Clin Endocrinol Metab 2020;105 (06): dgaa152

9 Park S, Kim WG, Song E, et al. Dynamic risk stratification for predicting recurrence in patients with differentiated thyroid cancer treated without radioactive iodine remnant ablation therapy. Thyroid 2017;27(04):524-530 